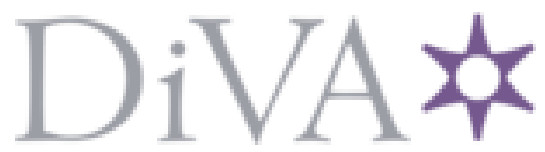

http://www.diva-portal.org

\title{
Postprint
}

This is the accepted version of a paper presented at General Meeting of the IEEE-Power-and-EnergySociety, JUL 26-30, 2015, Denver, CO.

Citation for the original published paper:

Dimoulkas, I., Amelin, M. (2015)

Probabilistic day-ahead CHP operation scheduling.

In: 2015 IEEE POWER \& ENERGY SOCIETY GENERAL MEETING IEEE

IEEE Power and Energy Society General Meeting PESGM

https://doi.org/10.1109/PESGM.2015.7285962

N.B. When citing this work, cite the original published paper.

Permanent link to this version:

http://urn.kb.se/resolve?urn=urn:nbn:se:kth:diva-184576 


\section{Probabilistic day-ahead CHP operation scheduling}

\author{
Ilias Dimoulkas \\ School of Electrical Engineering \\ Electric Power Systems \\ KTH Royal Institute of Technology \\ Stockholm, Sweden \\ Email: iliasd@kth.se
}

\author{
Mikael Amelin \\ School of Electrical Engineering \\ Electric Power Systems \\ KTH Royal Institute of Technology \\ Stockholm, Sweden \\ Email: amelin@kth.se
}

\begin{abstract}
The production scheduling of combined heat and power plants is a challenging task. The need for simultaneous production of heat and power in combination with the technical constraints results in a problem with high complexity. Furthermore, the operation in the electricity markets environment means that every decision is made with unknown electricity prices for the produced electric energy. In order to compensate the increased risk of operating under such uncertain conditions, tools like stochastic programming have been developed. In this paper, the short-term operation scheduling model of a CHP system in the day-ahead electricity market is mathematically described and solved. The problem is formulated in a stochastic programming framework where the uncertain parameters of dayahead electricity prices and the heat demand are incorporated into the problem in the form of scenarios. A case study is also performed with a CHP system operating in a district heating network and the value of heat storage capacity is estimated.
\end{abstract}

\section{INTRODUCTION}

The short-term operation scheduling of a system of power plants is a decision making problem regarding how much power and by which units is going to be produced during a short time period in the future, usually one day to one week ahead. This is a typical optimization problem, called unit commitment and economic dispatch, which is faced by power producers in order to minimize their costs and maximize their profits. In the special case of combined heat and power (CHP) systems this problem becomes more complex since the production of both power and heat need to be scheduled. Traditionally CHP systems find application in cases where there is a demand for both power and heat like industry and district heating. Small CHP systems, called micro CHP, are also used in commercial and residential buildings to provide heating and cooling. Compared to conventional power plants, CHP systems achieve a higher overall efficiency, resulting in reduced fuel consumption and exhaust gas emissions.

Various models have been proposed for the CHP shortterm production scheduling. The economic dispatch problem has been initially described and solved in [1], [2] and [3] where the problem is quadratic with linear constraints. In [4] a methodology based on dynamic programming and Lagrange relaxation is proposed. More recent works can be found in [5] and [6]. A comprehensive study of the various models and solutions that have been proposed for the CHP operation scheduling can be found in [7] and [8]. This paper describes a three-stage stochastic model with recourse which takes into account the variability of electricity prices and the heat demand. The model is similar to the two-stage model described in [9] with the addition of the possibility to store thermal energy and an extra stage in the decision tree. Furthermore, it is an extension of the model presented in [10] where the aim is to provide a bidding decision tool. Here instead, the aim is to provide a production scheduling tool.

The rest of the paper is organized as follows: section II provides the description and formulation of the problem. The decision framework is analyzed in subsection II-A while subsection II-B describes briefly the methodology used to generate and then reduce the stochastic parameter scenarios. The mathematical programming model is presented in subsection II-C. In section III a case study is performed where the value of the heat storage capacity is estimated. Finally section IV concludes the paper.

\section{Problem Formulation}

\section{A. The decision framework}

The formulation of the problem begins with the decisions that have to be made by the CHP plant operator. Such decisions may be the offering curves submitted to the electricity markets, the unit commitment and power dispatch scheduling, the heat dispatch for a CHP producer, the maintenance planning etc. Therefore during the first step the decision framework is determined and according to this the formulation of the model follows. Following the stochastic programming modeling framework, decision variables are split into two categories: the decisions that have to be made before any of the stochastic parameters have been realized (here-and-now) and decisions that are taken after the realization of some stochastic parameter (wait-and-see). This is the typical two stage model with recourse.

In this paper a three-stage model is proposed for the unit commitment and economic dispatch of a CHP system. The decision framework is as follows: after the clearing of the day ahead electricity market, the CHP operator is informed about the power assigned to produce during the following day (referred as Day-1 from now on). Therefore the unit commitment and power dispatch can be scheduled. These are the first stage decisions. The heat dispatch can be decided later, closer to the delivery hour and according to the heat load predictions that moment. Therefore, heat dispatch is the 
second stage decision and a number of heat load scenarios is used to take into account any possible heat load outcome. The third stage in the model is used to derive optimal unit commitment decisions for the terminal conditions, i.e. the final hours of Day-1. The coupling between power and heat production provides no obvious value for turning on or off the units at the end of Day-1 [9]. For that reason a third stage is added according to [9], where the decision variables are the power trading and heat dispatch during the next planning period (referred as Day-2 from now on). In that way, the optimal decision for unit commitment of the CHP system at the final hour of Day-1 takes into account all possible power and heat production variations during Day-2. The scenario tree of the proposed model is depicted in fig. 1.

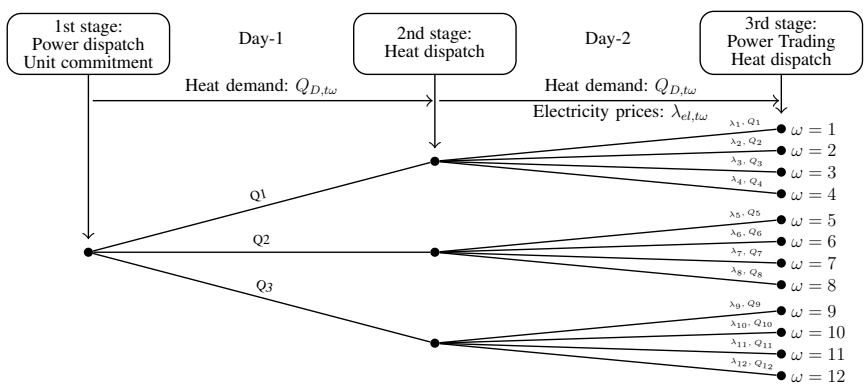

Figure 1. The 3-stage scenario tree of the proposed model

The model is used to derive optimal first stage decisions for the CHP operator in daily basis. The second stage decisions are also optimal for their respective scenarios. That means if Day1 's heat demand is exactly the same as a specific scenario, the optimal heat dispatch is given by the solution of the model for that scenario. If non of the scenarios is realized a rescheduling of the heat dispatch is done using the same model with fixed first stage decision variables to their optimal values.

\section{B. Scenario generation}

It has been described in II-A how variability of electricity prices and heat demand is taken into account by using scenarios of the specific parameters and constructing the scenario tree. Here, a methodology to generate the scenarios is briefly presented. This is done in two steps. First a time series model is created to forecast the uncertain parameter. A SARIMA model is used for the day-ahead electricity prices and a SARIMAX model for the heat demand where the external parameter is the outdoor temperature. This is justified by the fact that heat consumption and outdoor temperature is strongly negatively correlated in a district heating network as can be seen in fig. 2.

The second step is to use Monte Carlo simulation to produce the scenarios. Such scenarios for the day-ahead electricity prices can be seen in fig. 3. More details about the modeling procedure can be found in [10] and [11].

In order to approximate the distribution function of the stochastic parameters a great number of scenarios needs to be generated. This results in thousands of variables which makes the problem difficult to solve. For that reason a scenario reduction algorithm is applied in order to reduce the number

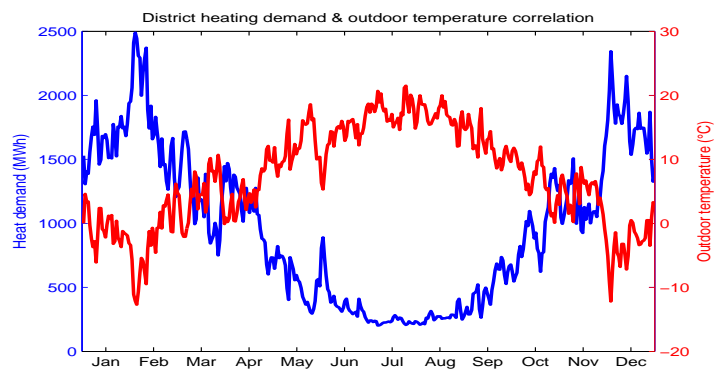

Figure 2. Yearly heat demand in Stockholm's district heating network and the outdoor temperature of the specific year

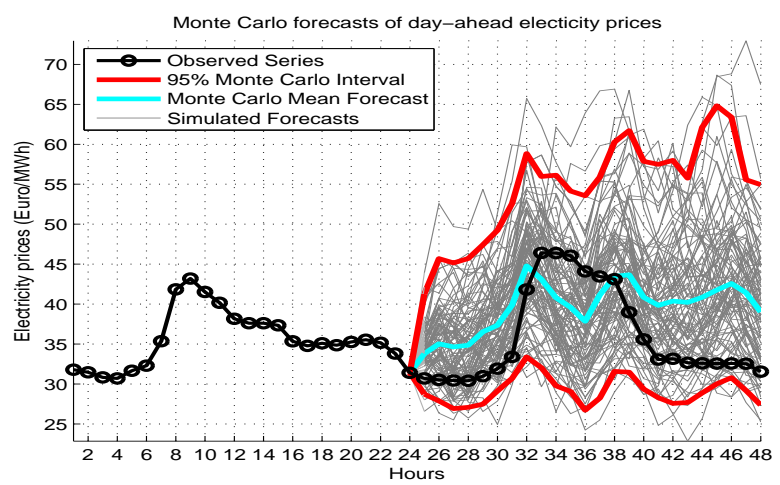

Figure 3. Day-ahead electricity price scenarios derived by Monte Carlo simulation

of the scenarios. The scenario reduction algorithm which is applied here is described in [11] and is based on minimizing the probability distance between the original set of scenarios and the simplified one. Finally, because heat demand and electricity prices are not correlated, all possible combinations are used to form the final scenario set.

\section{Mathematical formulation}

Objective function: The objective function (1) is to maximize the profits of the CHP producer which consist of the possible revenues from power sold during Day-2 (A), minus the variable production costs (B), the start-up costs (C) and the shut down costs (D) during both Day-1 and Day-2.

\section{Maximize :}

$$
\begin{aligned}
& \sum_{\omega=1}^{N_{\Omega}} \pi_{\omega}[\sum_{t=N_{T}+1}^{2 N_{T}} \sum_{g=1}^{N_{G}} \underbrace{\lambda_{e l, t \omega} P_{g t \omega}}_{A} \\
& \left.-\sum_{t=1}^{2 N_{T}} \sum_{g=1}^{N_{G}}(\underbrace{\lambda_{f, g} P_{f u e l, g t \omega}}_{B}+\underbrace{c_{\text {start }, g} y_{g t \omega}}_{C}+\underbrace{c_{\text {stop }, g} z_{g t \omega}}_{D})\right]
\end{aligned}
$$

Non-anticipativity constraints: These constraints (2-4) ensure that variables referred to common scenarios have to be assigned the same values. That means the first stage decisions 
will be the same for every scenario and the second stage decisions will be the same for common scenarios of Day-1.

$$
\begin{gathered}
P_{g t \omega}=P_{g t \omega+1}, \quad \forall g, t=1, \ldots, N_{T}, \omega=1, \ldots, N_{\Omega}-1 \\
u_{g t \omega}=u_{g t \omega+1}, \quad \forall g, t=1, \ldots, N_{T}, \omega=1, \ldots, N_{\Omega}-1 \\
Q_{g t \omega}=Q_{g t \omega+1}, \quad \forall g, t=1, \ldots, N_{T}, \omega=1, \ldots, N_{\Omega}-1: \\
\quad \text { if } Q_{D, t \omega}=Q_{D, t \omega+1}
\end{gathered}
$$

Operational constraints: The most common type of steam turbine used in large CHP systems is the extraction condensing steam turbine which is characterized for its flexibility. These plants can operate inside a feasible zone which is shown in fig. 4 and described by (5-8). The fuel consumption is given by (9). In many CHP plants there are heat producing boilers that are usually used during peak heat demand hours. The operational limits for these boilers are given by (10) and the fuel consumption by (11).

$$
\begin{aligned}
& \beta_{e l, g} P_{g t \omega}+\beta_{t h, g} Q_{g t \omega} \leqslant \beta_{e l, g} P_{\max , g} u_{g t \omega}, \quad \forall g, \forall t, \forall \omega \\
& \beta_{e l, g} P_{g t \omega}+\beta_{t h, g} Q_{g t \omega} \geqslant\left(\beta_{e l, g}+\beta_{t h, g} / r_{m i n, g}\right) P_{m i n, g} u_{g t \omega}, \\
& \forall g, \forall t, \forall \omega \\
& Q_{g t \omega} \leqslant Q_{\max , g}, \quad \forall g, \forall t, \forall \omega \\
& P_{g t \omega} \geqslant r_{\min , g} Q_{g t \omega}, \quad \forall g, \forall t, \forall \omega \\
& P_{f u e l, g t \omega}=\beta_{e l, g} P_{g t \omega}+\beta_{t h, g} Q_{g t \omega}+\beta_{0, g} u_{g t \omega}, \\
& \forall g, \forall t, \forall \omega \\
& Q_{\text {min, }, g} u_{g t \omega} \leqslant Q_{g t \omega} \leqslant Q_{\max , g} u_{g t \omega}, \quad \forall g, \forall t, \forall \omega \\
& P_{\text {fuel }, g t \omega}=\frac{Q_{g t \omega}}{\eta_{\text {boiler }, g}}, \quad \forall g, \forall t, \forall \omega
\end{aligned}
$$

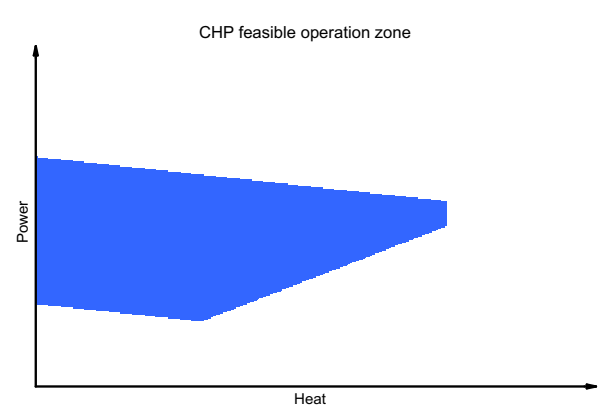

Figure 4. Feasible operation zone of an extraction condensing turbine

Heat balance constraints: The heat load balance constraint (12) ensures that the total heat production is equal to the total heat demand including the changes in the content of the heat storage tank. Constraint (13) restores the heat storage content at the end of Day-2 to its initial state. The capacity of the tank (14) limits the maximum heat content.

$$
\begin{gathered}
V_{t+1 \omega}=(1-a) V_{t \omega}+\sum_{g=1}^{N_{G}} Q_{g t \omega}-Q_{D, t \omega}, \\
V_{1 \omega}=(1-a) V_{2 N_{T} \omega}+\sum_{g=1}^{N_{G}} Q_{g 2 N_{T} \omega}-Q_{D, 2 N_{T} \omega}, \quad \forall \omega \\
V_{t \omega} \leqslant V_{\text {max }}, \quad \forall t, \forall \omega
\end{gathered}
$$

Power balance constraint: The power balance constraint (15) ensures that power output satisfies the load during Day-1.

$$
\sum_{g=1}^{N_{G}} P_{g t \omega}=P_{S, t}, \quad t=1, \ldots, N_{T}, \forall \omega
$$

Unit commitment constraints: These constraints (16-17) assign values to the binary variables $u, y$ and $z$ which keep the state of the units: operating, starting-up or shutting down respectively. With this formulation only variable $u$ needs to be stated as binary. The other two will automatically be assigned the right binary value.

$$
\begin{array}{r}
y_{g t \omega} \leqslant u_{g t \omega}, \quad y_{g t \omega} \leqslant 1-u_{g t-1 \omega}, \quad y_{g t \omega} \geqslant u_{g t \omega}-u_{g t-1 \omega}, \\
\forall g, \forall t, \forall \omega
\end{array}
$$

$$
\begin{array}{r}
z_{g t \omega} \leqslant u_{g t-1 \omega}, \quad z_{g t \omega} \leqslant 1-u_{g t \omega}, \quad z_{g t \omega} \geqslant u_{g t-1 \omega}-u_{g t \omega}, \\
\forall g, \forall t, \forall \omega
\end{array}
$$

Minimum up and down time constraints: These constraints (18-23) are applied to avoid the frequent transitions from on state to off state and vice versa.

$$
\begin{gathered}
\sum_{t=1}^{L_{g}}\left(1-u_{g t \omega}\right)=0, \quad \forall g, \forall \omega \\
\sum_{\tau=t}^{t+U T_{g}-1} u_{g \tau \omega} \geqslant U T_{g} y_{g t \omega}, \\
\forall g, t=L_{g}+1, \ldots, 2 N_{T}-U T_{g}+1, \forall \omega \\
\sum_{\tau=t}^{2 N_{T}}\left(u_{g \tau \omega}-y_{g t \omega}\right) \geqslant 0, \\
\quad \forall g, t=2 N_{T}-U T_{g}+2, \ldots, 2 N_{T}, \forall \omega \\
\sum_{t=1}^{F_{g}} u_{g t \omega}=0, \quad \forall g, \forall \omega \\
\left(1-u_{g \tau \omega}\right) \geqslant D T_{g} z_{g t \omega}, \quad \\
\sum_{\tau=t}^{t+D T_{g}-1} \quad \forall g, t=F_{g}+1, \ldots, 2 N_{T}-D T_{g}+1, \forall \omega \\
\sum_{\tau=t}^{2 N_{T}}\left(1-u_{g \tau \omega}-z_{g t \omega}\right) \geqslant 0, \\
\forall g, t=2 N_{T}-D T_{g}+2, \ldots, 2 N_{T}, \forall \omega
\end{gathered}
$$

where $L_{g}=\min \left\{2 N_{T},\left(U T_{g}-T_{u p, g}^{0}\right) u_{g}^{0}\right\}$ and $F_{g}=$ $\min \left\{2 N_{T},\left(D T_{g}-T_{\text {down }, g}^{0}\right)\left(1-u_{g}^{0}\right)\right\}$ are the hours in the beginning of the planning horizon that the unit is restricted to operate or to be offline respectively due to the initial conditions. 


\section{CASE STUDY}

In this section the results of a case study are presented. In this study a district heating network is considered. Two CHP plants provide hot water to the district heating system and sell the power to the day-ahead electricity market. An only heat boiler can be used when there is a peak on the heat demand. Furthermore a heat storage tank is available which can release or store hot water according to the schedule. The CHP system parameters are given in I. These parameters are mainly taken from [9] and [12]. District heating data used to produce the heat demand scenarios are from Stockholm's district heating network although they have been modified in order to fit this case study system. Electricity price scenarios are built with data from the Elspot market [13]. Random power load is chosen (taking values between 0-260 MW) in the form of 4-hour load blocks in order to resemble the power block offerings in day-ahead electricity market.

Table I

CHP PARAMETERS FOR THE CASE STUDY

\begin{tabular}{|c|c|c|}
\hline Extraction condensing steam turbine & Unit 1 & Unit 2 \\
\hline Fuel & \multicolumn{2}{|c|}{ Gas } \\
\hline Fuel price, $(€ / M W h)$ & \multicolumn{2}{|c|}{10} \\
\hline Min. power output, $(M W)$ & 35 & 30 \\
\hline Max. power output, $(M W)$ & 140 & 120 \\
\hline Max. heat output, $\left(M W_{t h}\right)$ & 200 & 180 \\
\hline Marginal fuel consumption for power production & 2.4 & 2.5 \\
\hline Marginal fuel consumption for heat production & 0.36 & 0.37 \\
\hline Fuel consumption at minimum output, $(M W)$ & 40 & 35 \\
\hline Minimum power-to-heat ratio & 0.5 & 0.5 \\
\hline Start-up cost, $(€)$ & 10000 & 10000 \\
\hline Shut down cost, $(€)$ & 0 & 0 \\
\hline Minimum up time, $(h)$ & 4 & 4 \\
\hline Minimum down time, $(h)$ & 3 & 3 \\
\hline Heat boiler & \multicolumn{2}{|c|}{ Unit 3} \\
\hline Fuel & \multicolumn{2}{|c|}{ Gas } \\
\hline Min. heat output, $\left(M W_{t h}\right)$ & \multicolumn{2}{|c|}{0} \\
\hline Max. heat output, $\left(M W_{t h}\right)$ & \multicolumn{2}{|c|}{80} \\
\hline Efficiency & \multicolumn{2}{|c|}{0.9} \\
\hline Start-up / Shut down cost, $(€)$ & \multicolumn{2}{|c|}{$0 / 0$} \\
\hline \multicolumn{3}{|l|}{ Heat storage tank } \\
\hline Capacity, $\left(M W h_{t h}\right)$ & \multicolumn{2}{|c|}{$0-1000$} \\
\hline Heat losses, (\%/hour) & \multicolumn{2}{|c|}{$0-2$} \\
\hline
\end{tabular}

In fig. 5 the unit commitment and power/heat production scheduling for the following day is presented for two cases. One for a system without any heat storage capacity and one with $600 \mathrm{MWh}_{\text {th }}$ capacity. Power production and unit commitment schedules as first stage decisions are optimal regardless of the scenarios. The heat production schedule depicted is for a random heat demand scenario and optimal only for that scenario as explained in II-A. According to the first case, the first CHP unit operates the whole day to provide the main part of the heat and power loads. This is the unit with the lower operational cost. The second CHP unit starts up when the first one is not sufficient to cover the power load and stops when this decreases again. For a few hours before the start up of the second unit and for one hour after, the heat boiler helps to cover the increased heat load. For that hours the first unit is sufficient to cover the power load alone. The increased flexibility of the system when a heat storage tank is used, can be seen in the second case. The system makes use of the stored hot water avoiding the use of the boiler and decreasing the operating hours of the second unit. For the hours that heat production is greater than heat demand, the charging of the heat storage tank takes place.
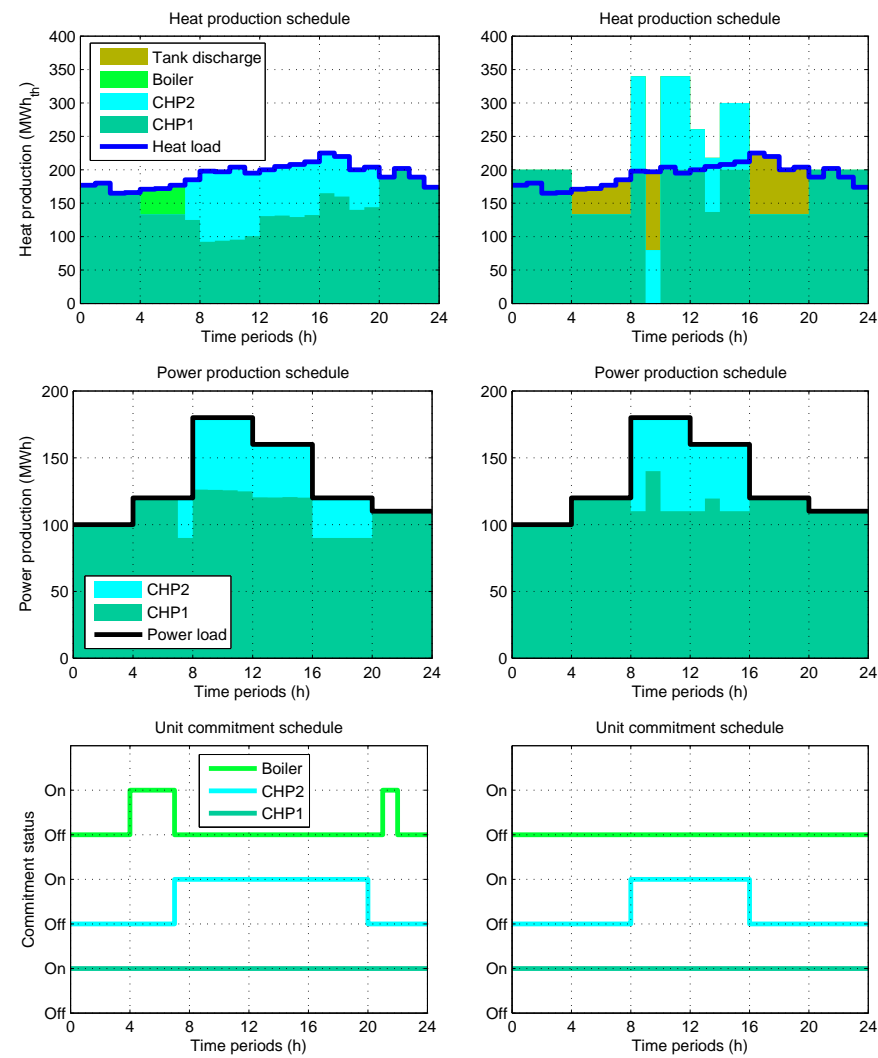

Figure 5. Next day's production scheduling and unit commitment of a system without (left column) and with (right column) heat storage capacity. Power production and unit commitment as first stage decisions are optimal. The heat production shown here is for a random heat load scenario of the scenario set and is optimal for the specific scenario.

To estimate the value of heat storage capacity in the case system, a yearly simulation of the system operation is conducted. The simulation is done with various heat storage capacities running from 0 to $1000 \mathrm{MWh}_{\mathrm{th}}$. Furthermore the impact of heat losses in the storage tank is studied by changing the heat loss parameter $\alpha$. Fig. 6 depicts the profit increase for various capacities compared to the system without any heat storage capacity. Profits are increasing in a logarithmic way reaching approx. 30\% for the case without any heat losses and $27 \%$ for the case with $2 \%$ per hour heat losses. The optimal heat storage capacity for a system can be estimated with a techno-economic analysis which will take into account the construction costs and the cost of money. Such analysis is beyond the scope of this paper. 


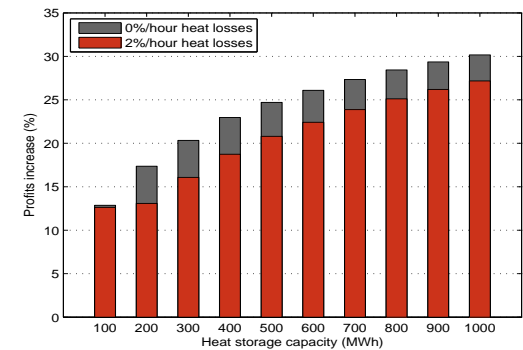

Figure 6. Profits increase for various heat storage capacities and heat losses

\section{CONCLUSIONS}

A MILP model is proposed for the short-term operation scheduling of a CHP system. Variable electricity prices and heat demand in a district heating network increase the risk of taking non optimal decisions. In order to compensate this uncertainty, stochastic programming framework is used to formulate the problem. Scenarios of the day-ahead electricity prices and the heat demand are built based on time series analysis and Monte Carlo simulation. A scenario reduction algorithm is also applied to decrease the size of the problem. The model provides the optimal day-ahead unit commitment and power/heat scheduling. As second stage variable, heat production can be rescheduled at a time closer to the realtime production if it is needed. The model also provides an estimation of the value of the heat storage capacity when it is run for a longer period. Up to $30 \%$ increase in profits can be achieved with big hot water storage tanks.

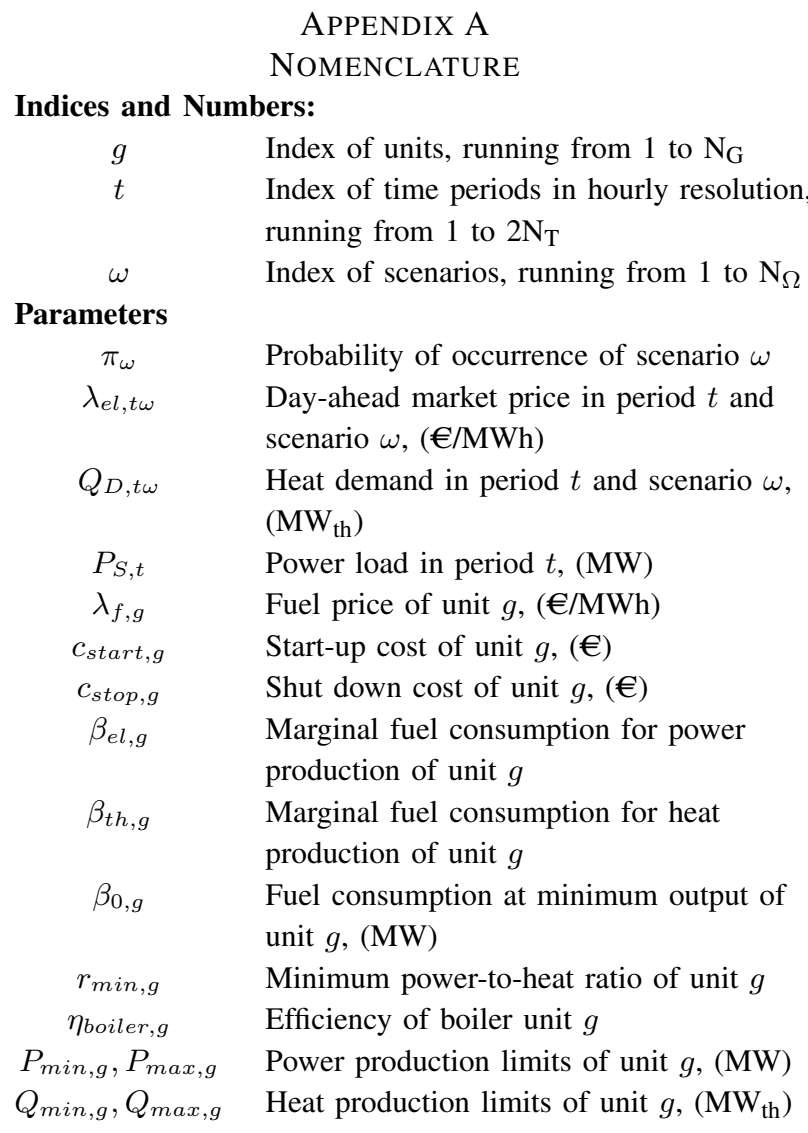

\begin{tabular}{|c|c|}
\hline$V_{\max }$ & Heat storage capacity, (MWh) \\
\hline$a$ & Heat storage losses, $(\% / \mathrm{h})$ \\
\hline$U T_{g}$ & Minimum up time of unit $g$, (h) \\
\hline$D T_{g}$ & Minimum down time of unit $g,(\mathrm{~h})$ \\
\hline$u_{g}^{0}, y_{g}^{0}, z_{g}^{0}$ & Initial state values \\
\hline$T_{u p, g}^{0}$ & $\begin{array}{l}\text { Time periods of unit } g \text { has been on in the } \\
\text { beginning of the planning horizon, }(\mathrm{h})\end{array}$ \\
\hline$T_{d o w n, g}^{0}$ & $\begin{array}{l}\text { Time periods of unit } g \text { has been off in the } \\
\text { beginning of the planning horizon, }(\mathrm{h})\end{array}$ \\
\hline \multicolumn{2}{|l|}{ Variables } \\
\hline$P_{g t \omega}$ & $\begin{array}{l}\text { Power produced by unit } g \text { in period } t \text { and } \\
\text { scenario } \omega,(\mathrm{MW})\end{array}$ \\
\hline$Q_{g t \omega}$ & $\begin{array}{l}\text { Heat produced by unit } g \text { in period } t \text { and } \\
\text { scenario } \omega,\left(\mathrm{MW}_{\mathrm{th}}\right)\end{array}$ \\
\hline$P_{f u e l, g t \omega}$ & $\begin{array}{l}\text { Fuel consumption of unit } g \text { in period } t \text { and } \\
\text { scenario } \omega,(\mathrm{MW})\end{array}$ \\
\hline$V_{t \omega}$ & $\begin{array}{l}\text { Heat storage content in period } t \text { and } \\
\text { scenario } \omega,(\mathrm{MWh})\end{array}$ \\
\hline$u_{g t \omega}$ & $\begin{array}{l}\text { Binary state variable for the on/off status of } \\
\text { unit } g \text { in period } t \text { and scenario } \omega\end{array}$ \\
\hline$y_{g t \omega}$ & $\begin{array}{l}\text { Sate variable for the start-up of unit } g \text { in period } \\
t \text { and scenario } \omega\end{array}$ \\
\hline$z_{g t \omega}$ & $\begin{array}{l}\text { State variable for the shut down of unit } g \text { in } \\
\text { period } t \text { and scenario } \omega\end{array}$ \\
\hline
\end{tabular}

\section{ACKNOWLEDGMENT}

This work was sponsored by SweGRIDS, the Swedish Centre for Smart Grids and Energy Storage, www.swegrids.se.

\section{REFERENCES}

[1] H. Puttgen and P. MacGregor, "Optimum scheduling procedure for cogenerating small power producing facilities," IEEE Transactions on Power Systems, vol. 4, no. 3, pp. 957-964, 1989.

[2] P. MacGregor and H. Puttgen, "A spot price based control mechanism for electric utility systems with small power producing facilities," IEEE Transactions on Power Systems, vol. 6, no. 2, pp. 683-690, 1991.

[3] F. Rooijers and R. A. M. Van Amerongen, "Static economic dispatch for co-generation systems," IEEE Transactions on Power Systems, vol. 9 , no. 3, pp. 1392-1398, 1994.

[4] E. Dotzauer, "Algorithms for short-term production-planning of cogeneration plants." Ph.D. dissertation, Division of Optimization, Department of Mathematics, Linköping University, Linköping, 1997.

[5] S. Groß, M. Rhein, and K. Rühling, "Large heat storages for load management in chp based district heating systems," Stockholm, 2014.

[6] M. Zugno, J. Morales, and H. Madsen, "Robust management of combined heat and power systems via linear decision rules," in Energy Conference (ENERGYCON), 2014 IEEE International, May 2014, pp. 479-486.

[7] F. Salgado and P. Pedrero, "Short-term operation planning on cogeneration systems: A survey," Electric Power Systems Research, vol. 78, no. 5 , pp. $835-848$, May 2008.

[8] A. Rong and R. Lahdelma, "Optimal operation of combined heat and power based power systems in liberalized power markets."

[9] C. Weber, Uncertainty in the electric power industry methods and models for decision support. New York: Springer, 2005.

[10] I. Dimoulkas and M. Amelin, "Constructing bidding curves for a CHP producer in day-ahead electricity markets," in Energy Conference (ENERGYCON), 2014 IEEE International, May 2014, pp. 487-494.

[11] A. J. Conejo, M. Carrión, and J. M. Morales, Decision Making Under Uncertainty in Electricity Markets, 2010th ed. Springer, Sep. 2010.

[12] C. Schaumburg-Müller, "Mathematical models and methods for analysis of distributed power generation on market conditions," Ph.D. dissertation, 2008.

[13] "Nord pool spot." [Online]. Available: http://www.nordpoolspot.com/ Market-data1/Elspot/Area-Prices/ALL1/Hourly/ 